\title{
Frühförderung: Überforderte Kinder - überforderte Erwachsene?
}

Wie lernen Kinder am besten? Werden sie später weniger erfolgreich, wenn sie nicht schon als Kleinkinder möglichst vielseitig gefördert werden? Überfordern Eltern ihre Kinder mit hohen Leistungsansprüchen - und auch sich selbst? Markus Opalka stellt Überlegungen dazu an und betrachtet neurowissenschaftliche Untersuchungen zum frühkindlichen Lernen näher.

Die Sonne scheint. Zusammen mit meinem Sohn Jo sitze ich auf einem abgemähten Kornfeld. Beide schauen wir in die Weite, kauen auf Grashalmen. Jo bestaunt den jagenden Greifvogel am Himmel, ist begeistert von dessen Flugkünsten im Wind. Wir sitzen da und tun nichts, mein Sohn und ich.

Momente wie diese werden seltener. Zeit und Natur einfach mal auf sich wirken lassen. Gemeinsame Augenblicke genießen, ohne Worte, ohne Absicht, ohne Verpflichtung. Aber nicht ohne Sinn! Wer leistet sich das schon noch. Terminkalender bestimmen nicht nur über die Erwachsenen, takten nicht nur unser Leben. Auch die Kinderwelt haben sie fest im Griff. Und Zeit ist längst nicht mehr nur in der Wirtschaft kostbar. Sie darf auch in der Erziehung und Ausbildung keinesfalls verschwendet werden. Das Gehirn, so glauben immer noch viele, funktioniert wie ein Muskelschwamm: Ständiges Training und alles mögliche aufsaugen, allein das bringt am Ende Erfolg.

\section{Bildung als Währung für die sichere Zukunft}

Genau das aber ist eine falsche Vorstellung, wie aktuelle Forschungsergebnisse aus der Neurowissenschaft zeigen. Der Neurobiologe Gerald Hüther brachte es im vorletzten Jahr in Berlin in seinem Eröffnungsvortrag anlässlich des Hauptstadtkongresses

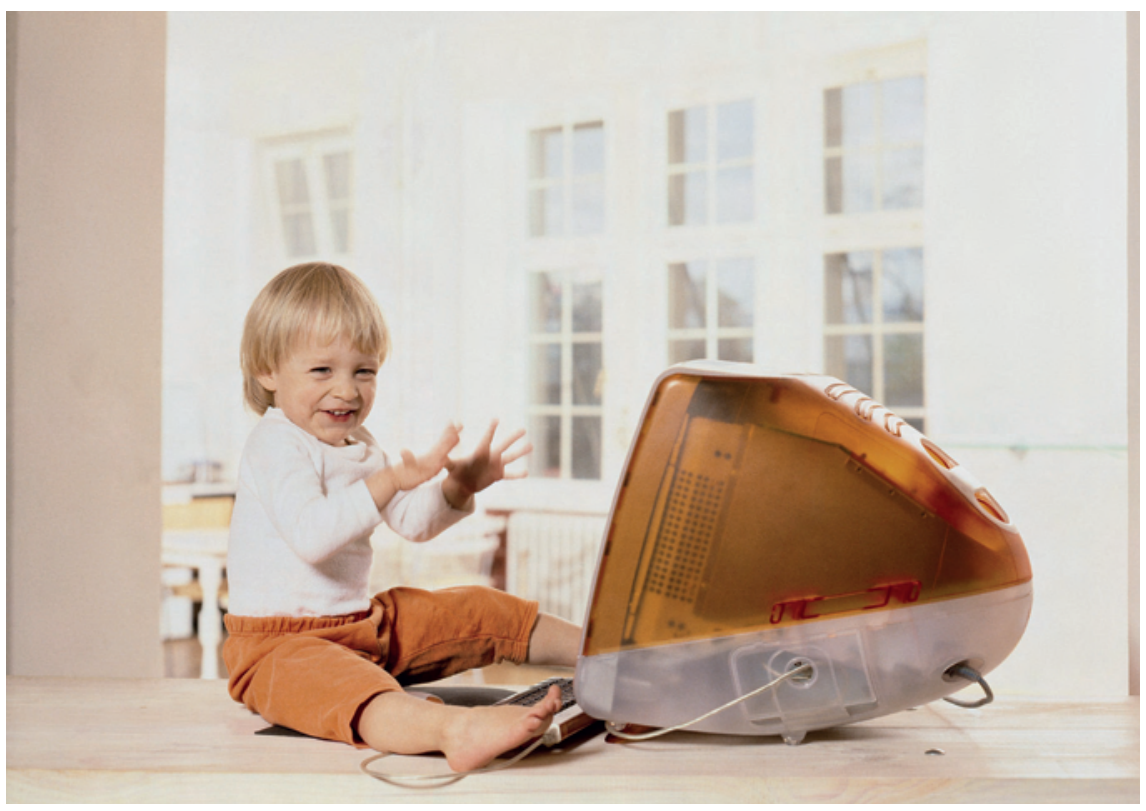

Abb. 1 Nicht die Menge an Frühförderunterricht macht's! Es muss das Richtige sein, denn Kinder lernen am besten mit Begeisterung. Foto: (c) MEV/Roland Bauer

„Gesundheit und Medizin“ auf den Punkt: „Das Gehirn wird nicht so, wie man es benutzt. Es wird so, wie man es mit Begeisterung nutzt!“

Und was machen Eltern mit ihren Kindern? Sie überfrachten sie mit Angeboten, die ihre Entwicklung fördern sollen: Pekipgruppen, Babyschwimmen, Kinderyoga, Musikwichtel, Chinesisch für Babys, Managerkurse für Kleinkinder. Neben Geigenunterricht, Frühspanisch und Hausaufgaben soll das das richtige Förderprogramm sein.

Und warum? Die Sorge um verpasste Chancen und Möglichkeiten ist es, die Eltern dazu treibt, ihren Nachwuchs unter Leistungsdruck zu setzen. Die Motive dafür sind unterschiedlich begründet: Die Kinder sollen es einmal besser haben als man es selbst hatte. Sie sollen den sozialen Aufstieg schaffen, den man vielleicht selbst nicht oder nur mit viel Mühe geschafft hat. Sie sollen auf alle Eventualitäten des Berufslebens vorbereitet sein.
Frühförderung ähnelt heute oft einem Wettrüsten und gipfelt vielfach in einer Leistungsschau unter Eltern. Da ergeben sich schon mal groteske Gespräche: „Wie? Laura kommt nicht mit zu den Musikwichteln?“ „Nein, Laura hat dafür keine Zeit. Laura besucht doch schon 2-mal in der Woche den Kurs Kreativmalen in der Stadt. Und beim Englisch müssen wir auch dranbleiben. Das wird dann zu viel!“ Übrigens: Laura ist 3.

Reden Eltern von Erziehung und Ausbildung, sprechen sie von Investitionen in die Zukunft. Sie deklarieren beides zum wirtschaftlichen Erfolgsmodell von morgen, die Währung heißt Bildung. Entwicklung darf nicht nur einfach geschehen. Sie unterliegt dem elterlichen und schulischen Controlling. Nichts soll dem Zufall überlassen sein. Alles ist kalkuliert, wird gesteuert.

Dagegen spricht allerdings ein bekanntes Sprichwort aus Afrika: „Das Gras wächst auch nicht schneller, wenn man daran zieht!“ 


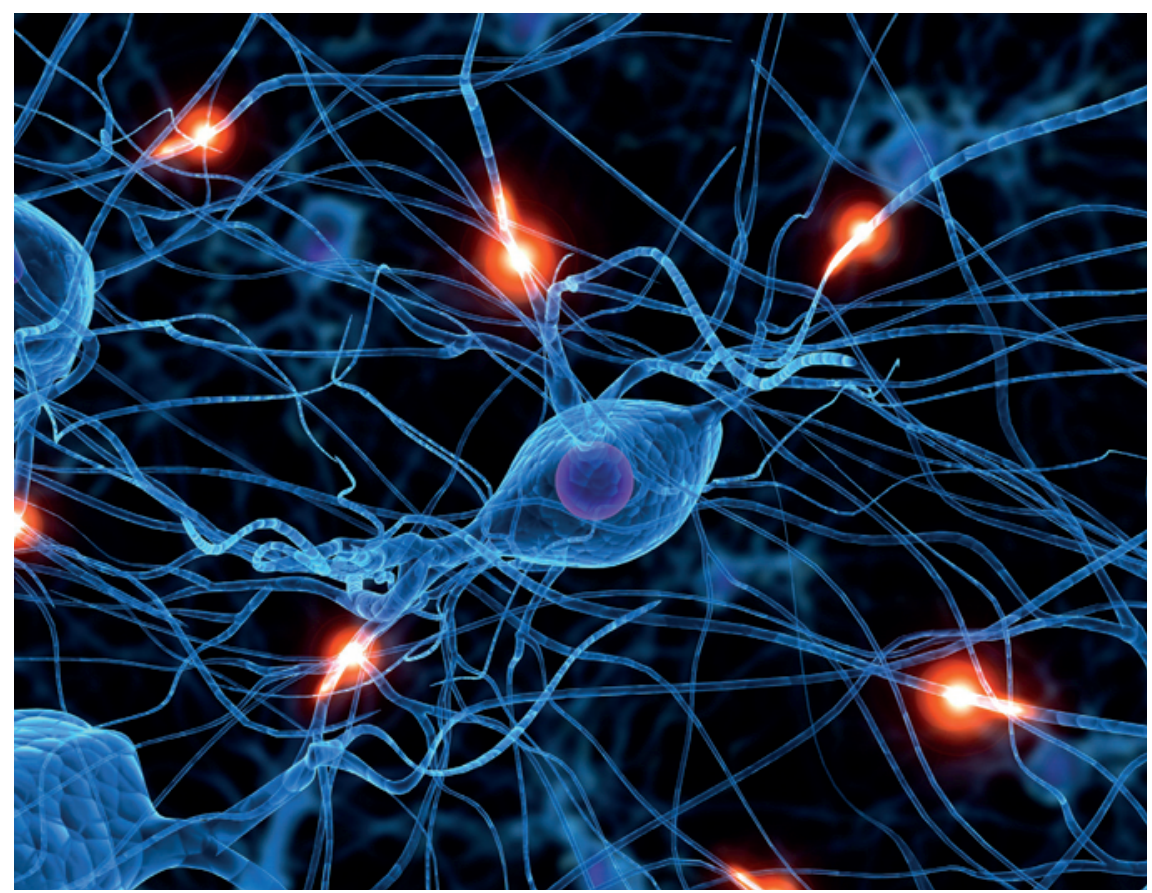

Abb. 2 Wenn ein Kind Spaß hat am Tun, mitgestalten kann und weiß, warum es etwas tut, sind die Voraussetzungen für eine neuronale Vernetzung optimal. Foto: @ Fotolia/ Sebastian Kaulitzki

\section{Stress an allen Fronten}

Den Leistungsdruck, den ihre Eltern initiieren, halten die wenigsten Kinder auf Dauer aus. Kinder kommen zunehmend mit psycho-physischen Beschwerden in Heilpraktiker- und Arztpraxen. Sie leiden unter Konzentrationsstörungen, Schlafproblemen oder Entwicklungsverzögerungen, sie fallen durch Hyperaktivität auf oder haben ständig Infekte. Die Universität Lüneburg hat in einer von der DAK beauftragten Studie herausgefunden, dass im Jahr 2010 bis zu 10 \% der 12- bis 17-Jährigen unter Depressionen litten. 30 \% der Schüler zeigten mehrmals pro Woche Stresssymptome wie Kopfschmerzen, Unlust, unregelmäßige Verdauung, Rückenschmerzen oder sogar klassische Entzugssymptome durch den frühzeitigen Genuss von stimulierenden Substanzen wie Kaffee, Nikotin oder Zucker.

Der Spagat zwischen erfolgreichem und selbstbestimmtem Berufsleben auf der einen und Familie auf der anderen Seite ist eine echte Herausforderung geworden. Und er gelingt nur wenigen. Viele Eltern schaffen ihn nicht ohne den rücksichtslosen Einsatz ihrer eigenen gesundheitlichen Ressourcen und auch der kindlichen. Und so kommen immer mehr Eltern mit den Kleinsten schon in die naturheilkundliche Praxis, weil aus ihren Kindern Schrei- kinder geworden sind oder ihre Babys keinen Schlaf mehr finden.

Früher gab es die Eltern und den Kinderarzt, wenn man Fragen hatte in puncto Kindererziehung oder Gesundheit seiner Kinder. Wer heute Fragen hat, verirrt sich in meterlangen Bücherregalen mit Ratgebern und Expertenwissen. Fernsehsendungen zeigen Lösungen für die schlimmsten Fälle. Mal ganz abgesehen von der Möglichkeit, nach jeder Lösung für individuelle und persönliche Probleme im World Wide Web zu googeln. Die Menge an Informationen bietet selten die ersehnte Antwort. Ganz im Gegenteil: Sie verschafft durch die Pluralität der angebotenen Lösungsmöglichkeiten eben keinen einfachen Konsens, sondern noch mehr Unsicherheit. Wie sollen Mütter und Väter da nicht verunsichert werden!

All das macht Stress. Letztlich nicht nur den Erwachsenen, sondern auch den Kindern.

\section{Begeistern kann nur gelingen}

Wir wollen alle nur das Beste für unsere Kinder. Das Beste aus unserer Sicht. Wir haben die Welt der Erwachsenen mit all ihren Ängsten und Bedürfnissen in das Kinderzimmer geholt. Und dort versuchen wir nun, unsere Kinder kompetent und fit zu machen für diese Welt. Mit allen Mitteln.
Dabei sollte uns etwas ganz anderes gelingen: Wir sollten das innere Kind mal wieder in die Welt der Erwachsenen bringen. Neugierde, Offenheit, einfach mal wieder etwas ausprobieren. Sich ganz zweckfrei für etwas begeistern können. Ohne Druck und Erfolgsgarantie. Aber mit der spannenden Aussicht, etwas Anderes, vielleicht auch etwas Bekanntes ganz neu zu entdecken.

Blicken wir einmal auf die Aussage des Neurobiologen Gerald Hüther zurück, so bleibt zu fragen: Welche Bedingungen müssen erfüllt sein, um Begeisterung und damit erfolgreiche Entwicklung zu ermöglichen? Was kennzeichnet also ein vernünftiges Setting von Förderangeboten?

Grundsätzlich, so Gerald Hüther, muss eine Bereitschaft zum Lernen vorhanden sein. Diese finden wir zweifelsfrei in der unbekümmerten Entdeckerleidenschaft eines jeden Kindes. Wichtig ist laut Hüther aber v. a. auch das Vertrauen. Vertrauen in einen offenen Lern- und Erfahrungsprozess mit folgenden Aspekten:

- Das Kind soll und kann das Geschehen verstehen.

- Es kann bewusst teilhaben und auch selbst aktiv mitgestalten.

- Vor allem aber soll das Kind einen persönlichen Sinn in dem entdecken, was es tut.

Das ist aber noch nicht alles: Erfolgreiche Entwicklung und damit Begeisterung kann man selbst dann noch nicht erzwingen. Sie kann nur gelingen! Wie beim Backen eines Kuchens: Wir haben alle nötigen Zutaten und vermischen sie nach Rezept, in der Hoffnung, dass der Kuchen durch das Backen gelingt. Aber es gelingt eben auch nicht immer.

Zudem muss sich neben dem kognitiven Anteil (z. B. frühes Sprachtraining) auch immer zeitgleich ein emotionaler Anteil im Gehirn aktivieren. Erst die Kombination aus beiden Anteilen lässt das Erlebte im Gehirn festschreiben. Bildhaft gesprochen: Jemand gießt mit einer großen Kanne Dünger über das Gehirn, emotionale und kognitive Areale im Gehirn schütten gleichzeitig neuroplastische Botenstoffe aus, die das aktuelle Erregungsmuster im Frontallappen des Gehirns festschreiben.

Für die frühkindliche Förderung heißt das ganz einfach: Hat ein Kind Freude bei dem, was es tut, versteht es, was es da 
eigentlich vollbringt und kann es selbst kreativ mitgestelten, dann haben wir ein Feuerwerk im Gehirn entfacht. Und Erfolg auf ganzer Linie!

\section{Entdecke die Möglichkeiten}

Bei all dem sind die Eltern gefordert! Denn gesundes und erfolgreiches Werden gelingt nur vor dem Hintergrund einer entsprechend gelebten geistigen Haltung, so Hüther. Es heißt ja auch Be-Geist-erung! Leben Eltern das vor, die Lust am Entdecken und Ausprobieren, und machen sie eben keine festen Vorgaben und haben keine vorgefertigten Vorstellungen, wie etwas zu werden hat, laden sie damit ihre Kinder ein, neue Erfahrungen zu sammeln. Sie ermutigen ihren Nachwuchs und inspirieren, etwas Neues zu wagen - mit der nötigen elterlichen Zuversicht und dem liebevollen Vertrauen in die Fähigkeiten des eigenen Kindes. Es entsteht Raum für freiheitliche Begegnung, in der das Interesse und die Begeisterung beim Kind für alles Mögliche geweckt werden können. Dabei steht diese
Haltung in keinster Weise der zeitweise nötigen Vorgabe entgegen, Grenzen zu setzen und auf Regeln im sozialen Miteinander hinzuweisen.

Gelingt es, unsere Kinder für Neues zu verzücken, dann schaffen wir die Grundlage für ein vernetztes und aktives Lernen. Wenn wir die Angebote aus dem täglichen Leben für unsere Kinder als eine lösbare Herausforderung gestalten, dann schaffen wir kreative Lösungswege, die sich in ihren Gehirnen festschreiben. Und schenken ihnen damit reichlich Potenzial für ihre $\mathrm{Zu}$ kunft.

Wenn wir in die Fähigkeiten und Kompetenzen unserer Kinder vertrauen und fest an sie glauben, dann ermutigen wir sie fortwährend. Eine gesunde Entwicklung sollte dann gelingen können - ohne Eltern und Kinder im Hier und Jetzt zu überfordern.

Dieser Artikel ist online zu finden unter: http://dx.doi.org//10.1055/s-0032-1326880

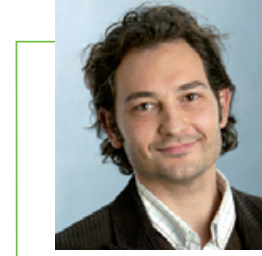

HP Markus Opalka MSc

Kleinherbeder Str. 9a 58455 Witten

Markus Opalka ist niedergelassen in eigener Naturheilpraxis in Witten (NRW). Als Heilpraktiker arbeitet er mit dem Schwerpunkt Osteopathie, insbesondere für Säuglinge/Kinder und mit Methoden der Regulationsmedizin.Darüber hinaus ist er als Dozent im Bereich Integrative Medizin im In- und Ausland tätig. Er arbeitet als Gesundheitswissenschaftler in weiteren Forschungsprojekten mit.Von 2006 bis Juli 2012 war er Mitherausgeber der DHZ.

E-Mail: post@markusopalka.de 